\title{
The trend malware source of IoT network
}

\author{
Susanto $^{1}$, M. Agus Syamsul Arifin ${ }^{2}$, Deris Stiawan ${ }^{3}$, Mohd. Yazid Idris ${ }^{4}$, Rahmat Budiarto \\ ${ }^{1,2}$ Faculty of Computer, Universitas Bina Insan, Indonesia \\ 1,2 Faculty of Engeenering, Universitas Sriwijaya, Indonesia \\ ${ }^{3}$ Faculty of Computer Science, Universitas Sriwijaya, Indonesia \\ ${ }^{4}$ Faculty of Computer Science and Information System, Universiti Teknologi Malaysia, Malaysia \\ ${ }^{5}$ College of Computer Science \& Information Technology, Albaha University, Al Baha, Saudi Arabia
}

\begin{tabular}{|c|c|}
\hline Article Info & ABSTRACT \\
\hline Article history: & \multirow{10}{*}{$\begin{array}{l}\text { Malware may disrupt the internet of thing (IoT) system/network when it } \\
\text { resides in the network, or even harm the network operation. Therefore, } \\
\text { malware detection in the IoT system/network becomes an important issue. } \\
\text { Research works related to the development of IoT malware detection have } \\
\text { been carried out with various methods and algorithms to increase detection } \\
\text { accuracy. The majority of papers on malware literature studies discuss } \\
\text { mobile networks, and very few consider malware on IoT networks. This } \\
\text { paper attempts to identify problems and issues in IoT malware detection } \\
\text { presents an analysis of each step in the malware detection as well as provides } \\
\text { alternative taxonomy of literature related to IoT malware detection. The } \\
\text { focuses of the discussions include malware repository dataset, feature } \\
\text { extraction methods, the detection method itself, and the output of each } \\
\text { conducted research. Furthermore, a comparison of malware classification } \\
\text { approaches accuracy used by researchers in detecting malware in IoT is } \\
\text { presented. }\end{array}$} \\
\hline Received Mar 19, 2020 & \\
\hline Revised Dec 5, 2020 & \\
\hline Accepted Jan 11, 2021 & \\
\hline Keywords: & \\
\hline Data repository malware & \\
\hline Feature extraction & \\
\hline IoT & \\
\hline Malware & \\
\hline Malware detection & \\
\hline
\end{tabular}

This is an open access article under the CC BY-SA license.

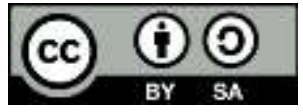

\section{Corresponding Author:}

Deris Stiawan

Faculty of Computer Science

Universitas Sriwijaya

Palembang, Indonesia

Email: deris@unsri.ac.id

\section{INTRODUCTION}

Internet of things (IoT) has different characteristics from other technologies that provide research opportunities in the study of malware in IoT. These characteristics are: 1) having an uncontrolled access environment where various devices connected to the IoT network are highly mobile. 2) heterogeneity where the diversity of devices interacting between devices that have high computing and those that have low-end computing such as servers with sensors and actuator devices. 3) scalability where the network on IoT devices is globally distributed but can be scaled in an application. 4) resource constraints where low energy requirements make the IoT design minimalist, so sensors and actuators limit security [1].

Malware or malicious software is a threat to information security and affects a computer system, a computer network, as well as cellular devices through the exploitation of system vulnerabilities [2]. Malware detection is a massive challenge at any time [3]. Malware detection is an action that must be prepared in the fight against attacks on IoT data security devices that were not designed during the initial stages of network development [4]. Malware may disrupt the IoT system/network when it resides in the network, or even harm the network operation. Therefore, malware detection in the IoT system/network becomes an important issue. Research works related to the development of IoT malware detection have been carried out with various 
methods and algorithms to increase detection accuracy. A malware detection system in IoT is a system that can recognize, even to find malware in a computer system, network traffic, node sensor packet data, in files, and inside the software, inside hardware, or an executable file installed on a computer system.

This paper attempts to identify problems and issues in IoT malware detection presents an analysis of each step in the malware detection as well as provides alternative taxonomy of literature related to IoT malware detection. The focuses of the discussions include malware repository dataset, feature extraction methods, the detection method itself, and the output of each conducted research.

The author of the paper provides an understanding of the evaluation methods of malware detection in IoT in addition to knowledge of data repositories, feature extraction, and detection methods. In particular, the study of malware literature on IoT is different from the study of malware literature on existing IoT, as listed in Table 1.

Table 1. Comparison of malware literature studies in IoT

\begin{tabular}{lcccc}
\hline \multirow{2}{*}{ Discussion Topics } & Karanja et al., 2017 [1] & Costin and Zaddah., 2018 [5] & Tahaei et al., 2020 [6] & Susanto et al., 2020 \\
\hline Data repository & - & $\sqrt{ }$ & $\sqrt{ }$ & $\sqrt{ }$ \\
Feature extraction & - & - & - & $\sqrt{ }$ \\
Detection Method & - & - & - & $\sqrt{ }$ \\
Output & - & - & - & $\sqrt{ }$ \\
\hline
\end{tabular}

\section{REVIEW OF LITERATURE}

\subsection{Data repository malware}

Malware detection is a part of the intrusion detection system (IDS). Research works on IoT malware detection use various datasets and traffic. Table 2 depicts a comparison of malware data sources versus evaluation methods used by researchers.

Authors of this paper observe from the results of a literature study that there are three types of malware source data used in IoT malware detection research. First, the use of malware captured directly from executable files, processors, or networks. The second one is the use of malware dataset. The third one is the use of malware captured from a testbed network.

Table 2. Comparison of data repository used by researchers

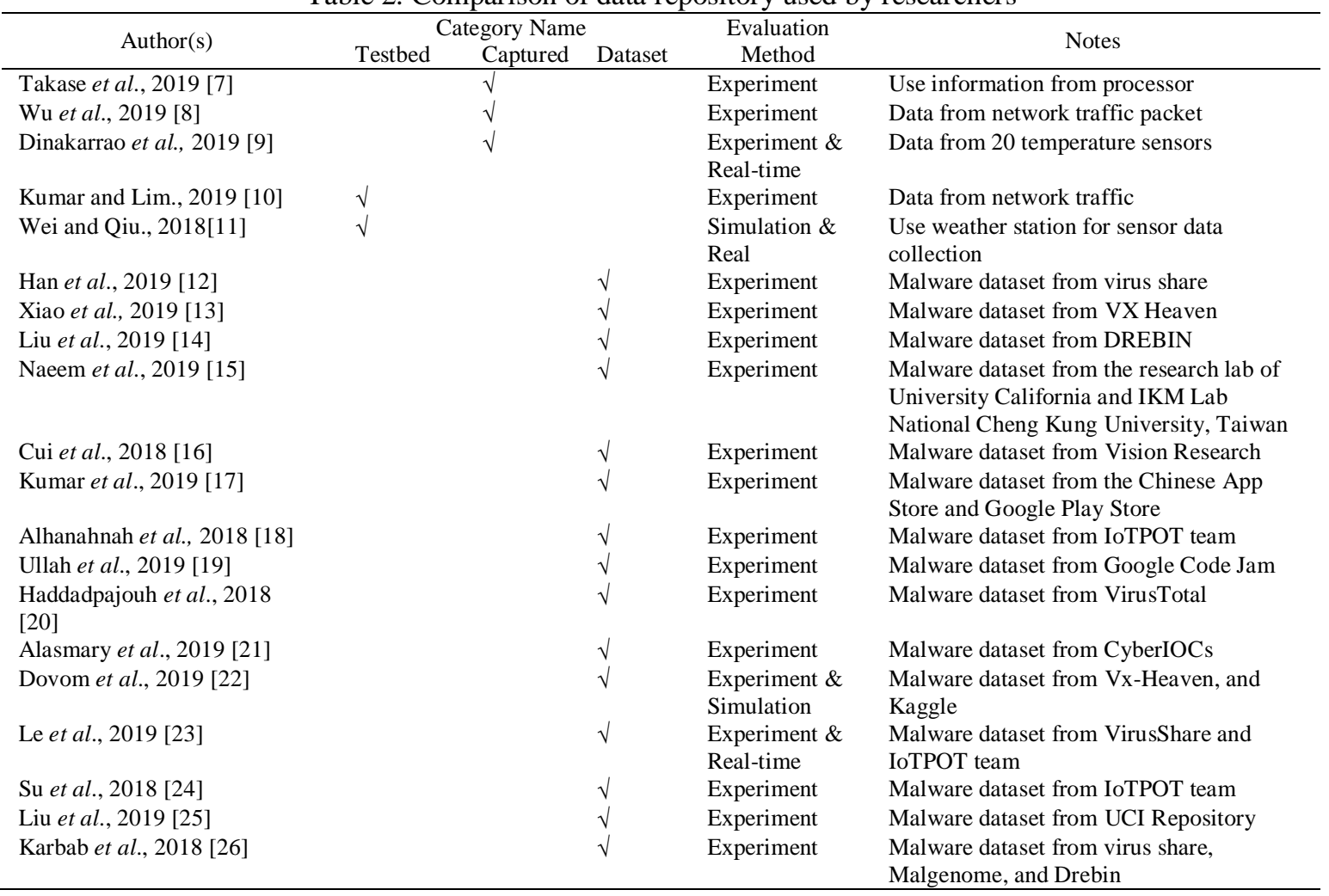


Table 2. Comparison of data repository used by researchers (continue)

\begin{tabular}{|c|c|c|c|c|c|}
\hline \multirow{2}{*}{ Author(s) } & \multicolumn{3}{|c|}{ Category Name } & \multirow{2}{*}{$\begin{array}{c}\text { Evaluation } \\
\text { Method }\end{array}$} & \multirow{2}{*}{ Notes } \\
\hline & Testbed & Captured & Dataset & & \\
\hline Nguyen et al., 2018 [27] & & & $\sqrt{ }$ & Experiment & Malware dataset from IoTPOT team \\
\hline Azmoodeh et al., 2018 [28] & & & $\sqrt{ }$ & Experiment & Malware dataset from VirusTotal \\
\hline Tzagkarakis et al., 2019 [29] & & & $\sqrt{ }$ & Experiment & Malware dataset from UCI Repository \\
\hline Dietz et al., 2018 [30] & $\sqrt{ }$ & & & $\begin{array}{l}\text { Experiment \& } \\
\text { Real-time }\end{array}$ & Data from the access router \\
\hline Meidan et at., 2018 [31] & & & $\sqrt{ }$ & Experiment & Malware dataset from UCI Repository \\
\hline McDermott et al., 2018 [32] & $\sqrt{ }$ & & & Experiment & Data from network traffic \\
\hline Bahsi et al., 2018 [33] & & & $\sqrt{ }$ & Experiment & Malware dataset from UCI Repository \\
\hline Abusnaina et al, 2019 [34] & & & $\sqrt{ }$ & Experiment & Malware dataset from CyberIOCs \\
\hline Manzanares et al., 2019 [35] & & & $\sqrt{ }$ & Experiment & $\begin{array}{l}\text { Malware dataset from UCI Repository } \\
\text { and Cyber Range Lab of UNSW } \\
\text { Canberra }\end{array}$ \\
\hline Namanya et al., 2019 [36] & & & $\sqrt{ }$ & Experiment & $\begin{array}{l}\text { Malware dataset from the repository of } \\
\text { Nettitude Ltd, UK }\end{array}$ \\
\hline Ham et al., 2014 [37] & & & $\sqrt{ }$ & Experiment & Malware dataset from Ham et al \\
\hline Ren et al., 2020 [38] & & & $\sqrt{ }$ & Experiment & $\begin{array}{l}\text { Malware dataset from VirusShare and } \\
\text { Google Play Store }\end{array}$ \\
\hline Nguyen et al., 2020 [39] & & & $\sqrt{ }$ & Experiment & $\begin{array}{l}\text { Malware dataset from VirusShare and } \\
\text { IOTPOT team }\end{array}$ \\
\hline Jung et al., 2020 [40] & & $\sqrt{ }$ & & Experiment & Data from power consumption \\
\hline
\end{tabular}

\subsection{Feature Extraction}

The first phase of malware detection is feature extraction. The extracted feature is initial information contains in an input file or resulted from an information processing [41]. The extraction process can be carried out using static analysis, dynamic analysis, and a combination of both [42-44]. A survey by researchers in [43] reports that static analysis consists of API calls, control flow graph (CFG), Opcode, and $\mathrm{N}$-gram; Dynamic analysis consists of function calls, function parameters, instruction traces, and instruction flow. S. Talukder [45] mention that static analysis consists of Opcode, N-gram, syntactic library, CFG, string signature, and others; dynamic analysis is a controlled environment such as virtual machines, simulators, emulators, sandboxes, and others. K. Diaz-Chito et al. [46] shows that the extraction process can also incremental. Furthermore, research work in [47] shows that the extraction process can also use deep learning. The feature extraction technique used in malware detection researches varies, some of them, as summarized in Table 3.

Table 3. Various feature extraction used in related researches

\begin{tabular}{|c|c|c|c|c|c|}
\hline Author(s) & $\begin{array}{c}\text { Static } \\
\text { Analysis }\end{array}$ & $\begin{array}{l}\text { Feature } \\
\text { Dynamic } \\
\text { Analysis }\end{array}$ & Other & Notes & Pros and contras \\
\hline $\begin{array}{l}\text { Takase et al., } \\
2019 \text { [7] }\end{array}$ & & Qemu & & $\begin{array}{l}\text { Extracting malware data from } \\
\text { CPU information }\end{array}$ & $\begin{array}{l}\text { Using an open-source emulator; } \\
\text { The information obtained is } \\
\text { incomplete if the source code is not } \\
\text { changed }\end{array}$ \\
\hline $\begin{array}{l}\text { Kumar and } \\
\text { Lim, } 2019 \\
{[10]}\end{array}$ & & & Feature vector & $\begin{array}{l}\text { Extract malware from a data } \\
\text { traffic packet }\end{array}$ & $\begin{array}{l}\text { Extraction results can be stored in } \\
\text { an online database }\end{array}$ \\
\hline $\begin{array}{l}\text { Xiao et al., } \\
2019 \text { [13] }\end{array}$ & API calls & $\begin{array}{l}\text { Cuckoo } \\
\text { Sandbox }\end{array}$ & $\begin{array}{l}\text { Stacked } \\
\text { AutoEncoders }\end{array}$ & $\begin{array}{l}\text { Extracting Portable } \\
\text { executable files }\end{array}$ & Can study malware behavior \\
\hline $\begin{array}{l}\text { Naeem, } 2019 \\
{[15]}\end{array}$ & & & $\begin{array}{l}\text { Deep } \\
\text { Convolutional } \\
\text { Neural Network }\end{array}$ & $\begin{array}{l}\text { Extracts executable malware } \\
\text { files into color images }\end{array}$ & $\begin{array}{l}\text { Can automatically extract malware; } \\
\text { The time needed for the extraction } \\
\text { process is faster }\end{array}$ \\
\hline $\begin{array}{l}\text { Cui et al., } \\
2018[16]\end{array}$ & & & $\begin{array}{l}\text { Convolutional } \\
\text { Neural Network }\end{array}$ & $\begin{array}{l}\text { Extracts executable malware } \\
\text { files into grayscale images }\end{array}$ & Can extract malware automatically \\
\hline $\begin{array}{l}\text { Kumar et al., } \\
2019 \text { [17] }\end{array}$ & & Dex2Jar & Blockchain & $\begin{array}{l}\text { Extracting executable .apk } \\
\text { files }\end{array}$ & $\begin{array}{l}\text { Faster and more accurate in } \\
\text { malware extraction }\end{array}$ \\
\hline $\begin{array}{l}\text { Alhanahnah } \\
\text { et al., } 2018 \\
{[18]}\end{array}$ & N-gram & Yara & & Extracting string feature & $\begin{array}{l}\text { Can execute word sequences on } \\
\text { unique IP addresses; }\end{array}$ \\
\hline $\begin{array}{l}\text { Ullah et al., } \\
2019[19]\end{array}$ & & & $\begin{array}{l}\text { Convolutional } \\
\text { Neural Network }\end{array}$ & $\begin{array}{l}\text { Extracts executable malware } \\
\text { files into color images }\end{array}$ & $\begin{array}{l}\text { Get a better visualization of } \\
\text { malware }\end{array}$ \\
\hline $\begin{array}{l}\text { Haddapajouh } \\
\text { et al., } 2018 \\
{[20]}\end{array}$ & $\begin{array}{l}\text { Opcode } \\
\text { and Object- } \\
\text { dump }\end{array}$ & & & $\begin{array}{l}\text { Extracting malware from } \\
\text { Debian package files }\end{array}$ & $\begin{array}{l}\text { Object-dump is only compatible } \\
\text { with Raspberry Pi II processors }\end{array}$ \\
\hline
\end{tabular}


Table 3. Various feature extraction used in related researches (continue)

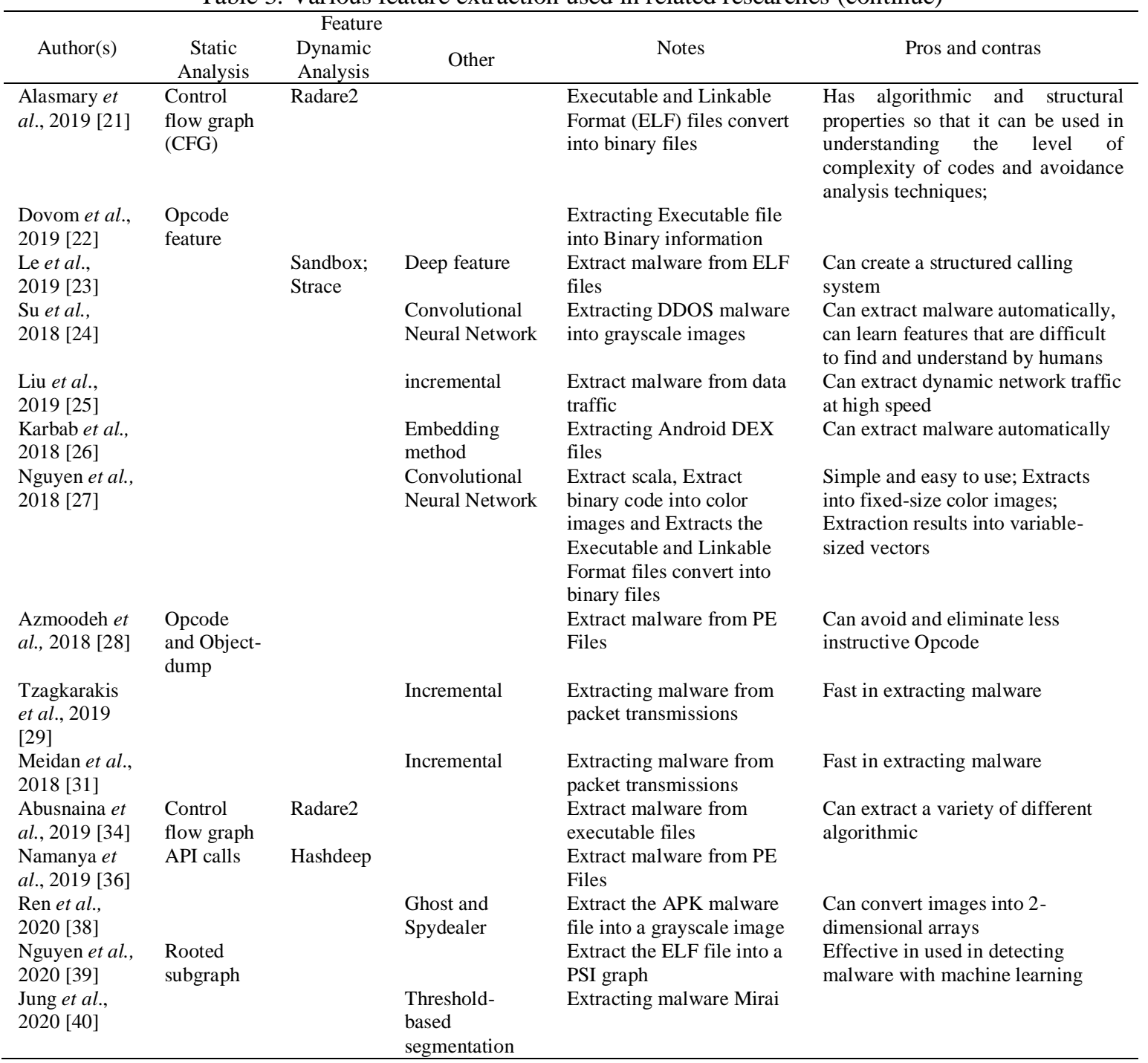

\subsection{Malware detection methods}

Various methods are used in malware detection research. A survey study by [48, 49] reveals that malware detection in IoT can use machine learning and deep learning methods. Another survey study by [50] says that malware detection in the CPU can use an emulator. Each method has advantages as well as disadvantages. A comprehensive study comparison of the use of malware detection methods was done by the author of this paper and summarized in Table 4.

Table 4. Comparison of the malware detection methods

\begin{tabular}{|c|c|c|c|c|}
\hline Author & Category & Methods/ Algorithm & Pros and cons & Accuracy \\
\hline $\begin{array}{l}\text { Takase et al., } \\
2019 \text { [7] }\end{array}$ & Emulator & Qети & High accuracy in malware detection. & $100 \%$ \\
\hline $\begin{array}{l}\text { Wu et al., } 2019 \\
{[8]}\end{array}$ & $\begin{array}{l}\text { Machine } \\
\text { learning }\end{array}$ & $\begin{array}{l}\text { Bayesian Model } \\
\text { Update Method }\end{array}$ & $\begin{array}{l}\text { Detecting malware based on traffic data. Having high } \\
\text { accuracy, ability to filter unuseful data or data having } \\
\text { negative impacts. The attribute must be independent }\end{array}$ & $96 \%$ \\
\hline $\begin{array}{l}\text { Dinakarrao et } \\
\text { al., 2019 [9] }\end{array}$ & $\begin{array}{l}\text { Machine } \\
\text { learning }\end{array}$ & OneR & $\begin{array}{l}\text { Detecting malware without creating overhead. If the } \\
\text { performance degrades under a threshold, then the } \\
\text { regulation process is stopped. Needing data in bulk }\end{array}$ & $92 \%$ \\
\hline $\begin{array}{l}\text { Kumar and } \\
\text { Lim., } 2019 \text { [10] }\end{array}$ & $\begin{array}{l}\text { Machine } \\
\text { learning }\end{array}$ & $\begin{array}{l}\text { Random Forest, } \\
\text { k-NN, Gaussian } \\
\text { Naïve Bayes }\end{array}$ & High accuracy in malware detection. & $\begin{aligned} \mathrm{RF} & =88.8 \% \\
\mathrm{k}-\mathrm{NN} & =94.44 \% \\
\mathrm{GNB} & =77.78 \%\end{aligned}$ \\
\hline $\begin{array}{l}\text { Wei and Qiu., } \\
2018 \text { [11] }\end{array}$ & Emulator & $\begin{array}{l}\text { Augmented Dickey- } \\
\text { Fuller test and } \\
\text { Mann-Kendall Test }\end{array}$ & Ability to know IoT devices that quickly infected & \\
\hline
\end{tabular}


Table 4. Comparison of the malware detection methods (continue)

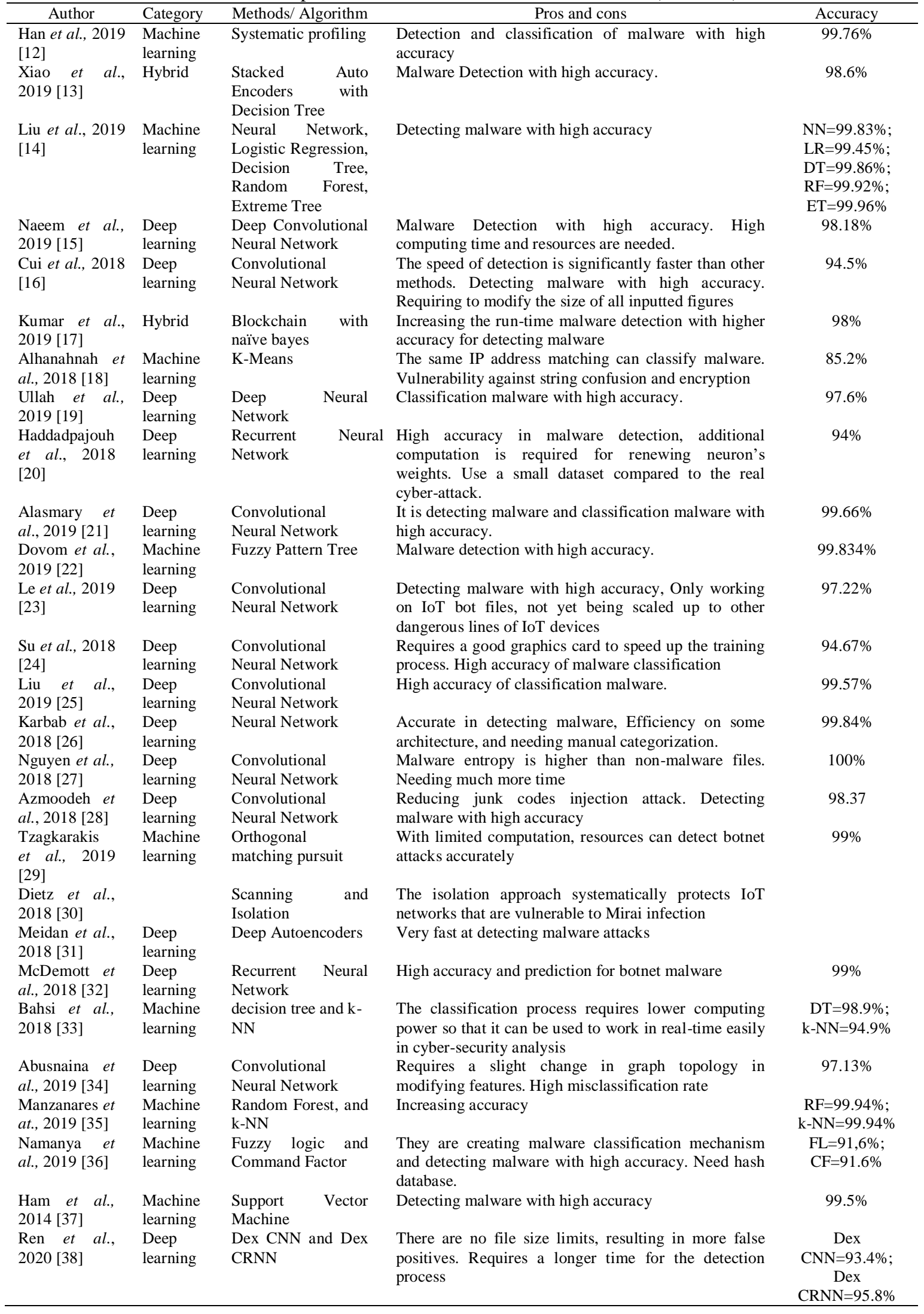


Table 4. Comparison of the malware detection methods (continue)

\begin{tabular}{llllc}
\hline \multicolumn{1}{c}{ Author } & Category & Methods/ Algorithm & \multicolumn{1}{c}{ Pros and cons } & Accuracy \\
\hline Nguyen et al., & Machine & Support Vector & The use of rooted subgraph extraction features with & SVM= 99.6\%; \\
$2020[39]$ & learning & $\begin{array}{l}\text { Machine, Decision } \\
\text { Tree, k-NN, Random } \\
\text { machine learning results in better detection accuracy. } \\
\text { Bagging=976\%; } \\
\text { Forest, and Bagging }\end{array}$ & Allow for errors in the storage of all subgraphs & DT=98.7\%; \\
RF=991\%; & k-NN=99.2\% \\
Jung et al., & Deep & $\begin{array}{l}\text { Convolutional } \\
\text { Ne20 [40] }\end{array}$ & $\begin{array}{l}\text { It is detecting malware with high accuracy. It cannot be } \\
\text { integrated on IoT devices. }\end{array}$ & $98.6 \%$ \\
\hline
\end{tabular}

\subsection{Output}

Overall, the output of the existing IoT malware detection researches is in the form of scores and labels. The score is the output of every trial in the experiment in the form of detection accuracy rankings. Research in [22] produces classification accuracy in terms of the highest rank. The label is the output from every experimental trial in the form of label 'malware' or 'benign.' Research in [14] produces output from detecting malware in the form of malware label and benign label. Research in [25] considers the output is in the form of benign traffic label and attack traffic label. Research in [7] produces output in the form of a normal label and attack label.

\section{DISCUSSION AND ANALYSIS}

Literature shows that in IoT malware detection researches, the malware data repository (dataset) is taken from testbed, self capturing, and various public dataset sources. Table 2 presents data repositories that have been used in researches that show $76.47 \%$ using malware dataset, $11.76 \%$ using malware captured directly from processor and network, and $11.76 \%$ using the testbed network. The most used public dataset is sourced from IoTPOT of Yokohama National University. The dataset is labeled by two types: malware and benign. From the data repositories used by researchers, the majority of IoT malware detection research is mostly only done as an experiment in a laboratory. It is not done in a real-time fashion so that it becomes a challenge on how to implement IoT malware detection in real-time. IoT technology has different characteristics, so that it has a more significant problem in detecting malware in real-time. The first challenge is developing a fast and lightweight detection system without using huge costs [9]. Second, developing energy-efficient detection systems with limited resources [18], and the third one is identifying known malware and new malware in real cyberattacks using a small dataset at the time of the experiment [20].

In extracting the information from the dataset and then in the classification, data in Table 3 presents feature extraction consisting of static analysis, dynamic analysis, and also a combination of static and dynamic analysis. Also, there is a feature extraction using incremental, deep learning, and blockchain. Attributes in the static analysis that have been used by researchers include API calls, N-grams, Opcodes, Control flow graph, rooted subgraphs. There are also those using open-source Object-dump tools, while in dynamic analysis, the tools that have been used by researchers in the form of open-source tools include Cuckoo Sandbox, Dex2Jar, Yara, Qemu, Radare2, Object-dump, Strace, hashdeep. Each malware analysis tool can be used to extract different malware files. From the results of the literature studies, extraction feature is used to extract malware from network traffic, executable files, and processors. The feature extraction method that is most widely used by researchers in deep learning. By using deep learning, the features can automatically be extracted [15, 16, 24], and be able to learn on its own from the malware [13].

Data in Table 4 presents the malware detection methods on IoT. The information on the detection methods from literature is divided into three categories, namely machine learning, deep learning, and emulator. Machine learning methods that have been used by researchers include logistic regression, Decision trees, random forests, extreme trees, k-means, fuzzy pattern trees, fuzzy logic, orthogonal matching pursuit, support vector machines, k-nearest neighbors, and Bagging. In contrast, in deep learning, the methods that have been used by researchers include neural networks, convolutional neural networks, deep neural networks, deep convolutional neural networks, recurrent neural networks, deep autoencoders, Dex CNN and Dex CRNN. Besides, researchers also used the Qemu emulator and the augmented Dickey-Fuller test and the Mann-Kendall test. Then there are also researchers with hybrid methods, including neural network stacked auto encoders with decision tree and blockchain with naive Bayes. Machine learning and deep learning are used to perform binary classification, i.e., to classify whether the application file is a malware or not. From the results of literature studies, the most widely used malware detection method is deep learning with the convolutional neural network algorithm. The convolutional neural network algorithm requires a good graphics card to speed up the training process [24]. Decision tree, Orthogonal matching pursuit, and k-NN in the classification process require lower computing power so that it can be used to work in real-time 
efficiently in the analysis of malware attacks on IoT [33]. The output is a final result of malware detection with the majority in the form of labels (malware and benign).

There are several indicators used in measuring the performance of classification accuracy, from the use of malware repository data, feature extraction to malware classification methods. The indicators used in each study differ from each other, and some papers do not address the issue of detection accuracy. In this paper, the authors present the results of a literature review paper on malware detection on IoT by comparing the accuracy of each approach used by researchers, as shown in Table 4 . The results of the study presented in Table 4 have an average high level of detection accuracy.

Furthermore, we analyze literature that contributes to IoT malware detection researches. The IoT networks have different characteristics so that it becomes a challenge in malware detection. Data acquisition from sensors, Android devices, and network protocols should be extracted using the appropriate method with the primary aim that the information of the data can be read. The information yielded from the extraction process will then be analyzed to determine whether the data packet is malware or benign. In some cases, there are traffic data that are not recognized, so they need an algorithm that can identify those data using a smart/intelligent system automatically. Therefore, the feature extraction and method in IoT malware detection become the primary key to the success of malware detection.

\section{CONCLUSION AND FUTURE WORK}

An alternative taxonomy of literature related to IoT malware detection has been discussed. The focuses of the discussions include malware repository dataset, feature extraction methods, the detection method itself, and the output of each conducted research. In conducting malware detection experiments on IoT, input data may use self captured data, testbed as well as public datasets. Several datasets for malware detection on IoT has been provided by researchers and are ready to be used for research according to the selected scenario. Feature extraction is one of the crucial processes in malware detection. Extracting malware features may use static or dynamic methods or a combination of both, even combining with the use of deep learning features. The dynamic methods can be implemented using open source tools. Each feature extraction has advantages and disadvantages of each. The classification method is used to determine the output of malware detection, whether the data is malware or not. From the classified output, the level of accuracy of the detection can be measured. Besides, this paper has analyzed each step of IoT malware detection. The alternative taxonomy complements existing literature studies, strips issues of malware detection in IoT network/system, and helps researchers in designing reliable malware detection system for IoT network/system. Real-time IoT malware detection system development is considered one of the future works in this research area.

\section{REFERENCES}

[1] E. M. Karanja, S. Masupe, and J. Mandu, "Internet of Things Malware : A Survey," Int. J. Comput. Sci. Eng. Surv., vol. 8, no. 3, pp. 1-20, 2017, doi: 10.5121/ijcses.2017.8301.

[2] A. O. Eze and C. C. E, "Malware Analysis and Mitigation in Information Preservation," IOSR J. Comput. Eng., vol. 20, no. 4, pp. 53-62, 2018, doi: 10.9790/0661-2004015362.

[3] M. Akour, I. Alsmadi, and M. Alazab, "The malware detection challenge of accuracy," 2016 2nd Int. Conf. Open Source Softw. Comput. OSSCOM 2016, 2017, doi: 10.1109/OSSCOM.2016.7863750.

[4] C. Vorakulpipat, E. Rattanalerdnusorn, P. Thaenkaew, and H. Dang Hai, "Recent challenges, trends, and concerns related to IoT security: An evolutionary study," Int. Conf. Adv. Commun. Technol. ICACT, vol. 2018-Febru, pp. 405-410, 2018, doi: 10.23919/ICACT.2018.8323774.

[5] A. Costin and J. Zaddach, "IoT Malware: Comprehensive Survey, Analysis Framework and Case Studies," BlackHat 2018 USA, 2018.

[6] H. Tahaei, F. Afifi, A. Asemi, F. Zaki, and N. B. Anuar, "The rise of traffic classification in IoT networks: A survey," J. Netw. Comput. Appl., vol. 154, p. 102538, 2020, doi: 10.1016/j.jnca.2020.102538.

[7] H. Takase, R. Kobayashi, M. Kato, and R. Ohmura, "A prototype implementation and evaluation of the malware detection mechanism for IoT devices using the processor information," Int. J. Inf. Secur., 2019, doi: 10.1007/s10207-019-00437-y.

[8] F. Wu, L. Xiao, and J. Zhu, "Bayesian Model Updating Method Based Android Malware Detection for IoT Services," 2019 15th Int. Wirel. Commun. Mob. Comput. Conf., pp. 61-66, 2019, doi: 10.1109/iwcmc.2019.8766754.

[9] S. M. Pudukotai Dinakarrao, H. Sayadi, H. M. Makrani, C. Nowzari, S. Rafatirad, and H. Homayoun, "Lightweight Node-level Malware Detection and Network-level Malware Confinement in IoT Networks," Proc. 2019 Des. Autom. Test Eur. Conf. Exhib. DATE 2019, pp. 776-781, 2019, doi: 10.23919/DATE.2019.8715057.

[10] A. Kumar and T. J. Lim, "EDIMA: Early Detection of IoT Malware Network Activity Using Machine Learning Techniques,” 2019 IEEE 5th World Forum Internet Things, pp. 289-294, 2019, doi: 10.1109/wf-iot.2019.8767194. 
[11] D. Wei and X. Qiu, "Status-based Detection of malicious code in Internet of Things (IoT) devices," 2018 IEEE Conf. Commun. Netw. Secur. CNS 2018, pp. 1-7, 2018, doi: 10.1109/CNS.2018.8433183.

[12] W. Han, J. Xue, Y. Wang, Z. Liu, and Z. Kong, "MalInsight: A systematic profiling based malware detection framework," J. Netw. Comput. Appl., vol. 125, pp. 236-250, 2019, doi: 10.1016/j.jnca.2018.10.022.

[13] F. Xiao, Z. Lin, Y. Sun, and Y. Ma, "Malware Detection Based on Deep Learning of Behavior Graphs," Math. Probl. Eng., vol. 2019, 2019, doi: 10.1155/2019/8195395.

[14] X. Liu, X. Du, X. Zhang, Q. Zhu, H. Wang, and M. Guizani, "Adversarial samples on android malware detection systems for IoT systems,” Sensors (Switzerland), vol. 19, no. 4, pp. 1-16, 2019, doi: 10.3390/s19040974.

[15] H. Naeem, "Detection of Malicious Activities in Internet of Things Environment Based on Binary Visualization and Machine Intelligence," Wirel. Pers. Commun., vol. 108, no. 4, pp. 2609-2629, 2019, doi: 10.1007/s11277-01906540-6.

[16] Z. Cui, F. Xue, X. Cai, Y. Cao, G. G. Wang, and J. Chen, "Detection of Malicious Code Variants Based on Deep Learning," IEEE Trans. Ind. Informatics, vol. 14, no. 7, pp. 3187-3196, 2018, doi: 10.1109/TII.2018.2822680.

[17] R. Kumar, X. Zhang, W. Wang, R. U. Khan, J. Kumar, and A. Sharif, "A Multimodal Malware Detection Technique for Android IoT Devices Using Various Features,” IEEE Access, vol. 7, no. May, pp. 64411-64430, 2019, doi: 10.1109/ACCESS.2019.2916886.

[18] M. Alhanahnah, Q. Lin, Q. Yan, N. Zhang, and Z. Chen, "Efficient signature generation for classifying cross-architecture IoT malware," 2018 IEEE Conf. Commun. Netw. Secur. CNS 2018, pp. 1-9, 2018, doi: 10.1109/CNS.2018.8433203.

[19] F. Ullah et al., "Cyber Security Threats Detection in Internet of Things Using Deep Learning Approach," IEEE Access, vol. 7, pp. 124379-124389, 2019, doi: 10.1109/access.2019.2937347.

[20] H. HaddadPajouh, A. Dehghantanha, R. Khayami, and K. K. R. Choo, "A deep Recurrent Neural Network based approach for Internet of Things malware threat hunting," Futur. Gener. Comput. Syst., vol. 85, pp. 88-96, 2018, doi: 10.1016/j.future.2018.03.007.

[21] H. Alasmary et al., "Analyzing and Detecting Emerging Internet of Things Malware: A Graph-based Approach," IEEE Internet Things J., vol. 4662, no. c, pp. 1-1, 2019, doi: 10.1109/jiot.2019.2925929.

[22] E. M. Dovom, A. Azmoodeh, A. Dehghantanha, D. E. Newton, R. M. Parizi, and H. Karimipour, "Fuzzy pattern tree for edge malware detection and categorization in IoT," J. Syst. Archit., vol. 97, pp. 1-7, 2019, doi: 10.1016/j.sysarc.2019.01.017.

[23] H. Le, Q. Ngo, and V. Le, "Iot Botnet Detection Using System Call Graphs and One-Class CNN Classification," Int. J. Innov. Technol. Explor. Eng., vol. 8, no. 10, pp. 937-942, 2019, doi: 10.35940/ijitee.j9091.0881019.

[24] J. Su, V. Danilo Vasconcellos, S. Prasad, S. Daniele, Y. Feng, and K. Sakurai, "Lightweight Classification of IoT Malware Based on Image Recognition,” Proc. - Int. Comput. Softw. Appl. Conf., vol. 2, pp. 664-669, 2018, doi: 10.1109/COMPSAC.2018.10315.

[25] J. Liu, S. Liu, and S. Zhang, "Detection of IoT botnet based on deep learning," Chinese Control Conf. CCC, vol. 2019-July, no. 1, pp. 8381-8385, 2019, doi: 10.23919/ChiCC.2019.8866088.

[26] E. M. B. Karbab, M. Debbabi, A. Derhab, and D. Mouheb, "MalDozer: Automatic framework for android malware detection using deep learning," DFRWS 2018 EU - Proc. 5th Annu. DFRWS Eur., vol. 24, pp. S48-S59, 2018, doi: 10.1016/j.diin.2018.01.007.

[27] K. D. T. Nguyen, T. M. Tuan, S. H. Le, A. P. Viet, M. Ogawa, and N. Le Minh, "Comparison of Three Deep Learning-based Approaches for IoT Malware Detection," Proc. 2018 10th Int. Conf. Knowl. Syst. Eng. KSE 2018, pp. 382-387, 2018, doi: 10.1109/KSE.2018.8573374.

[28] A. Azmoodeh, A. Dehghantanha, and K.-K. R. Choo, "Robust Malware Detection for Internet of (Battlefield) Things Devices Using Deep Eigenspace Learning," IEEE Trans. Sustain. Comput., vol. 4, no. 1, pp. 88-95, 2018, doi: 10.1109/tsusc.2018.2809665.

[29] C. Tzagkarakis, N. Petroulakis, and S. Ioannidis, "Botnet attack detection at the IoT edge based on sparse representation," Glob. IoT Summit, GIoTS 2019 - Proc., pp. 1-6, 2019, doi: 10.1109/GIOTS.2019.8766388.

[30] C. Dietz et al., "IoT-Botnet Detection and Isolation by Access Routers," Proc. 2018 9th Int. Conf. Netw. Futur. NOF 2018, pp. 88-95, 2018, doi: 10.1109/NOF.2018.8598138.

[31] Y. Meidan et al., "N-BaIoT-Network-based detection of IoT botnet attacks using deep autoencoders," IEEE Pervasive Comput., vol. 17, no. 3, pp. 12-22, 2018, doi: 10.1109/MPRV.2018.03367731.

[32] C. D. McDermott, F. Majdani, and A. V. Petrovski, "Botnet Detection in the Internet of Things using Deep Learning Approaches," Proc. Int. Jt. Conf. Neural Networks, vol. 2018-July, pp. 1-8, 2018, doi: 10.1109/IJCNN.2018.8489489.

[33] H. Bahsi, S. Nomm, and F. B. La Torre, "Dimensionality Reduction for Machine Learning Based IoT Botnet Detection," 2018 15th Int. Conf. Control. Autom. Robot. Vision, ICARCV 2018, pp. 1857-1862, 2018, doi: 10.1109/ICARCV.2018.8581205.

[34] A. Abusnaina et al., "Examining Adversarial Learning against Graph-based IoT Malware Detection Systems," 2019.

[35] A. Guerra-Manzanares, H. Bahsi, and S. Nomm, "Hybrid feature selection models for machine learning based botnet detection in IoT networks," Proc. - 2019 Int. Conf. Cyberworlds, CW 2019, pp. 324-327, 2019, doi: 10.1109/CW.2019.00059.

[36] A. P. Namanya, I. U. Awan, J. P. Disso, and M. Younas, "Similarity hash based scoring of portable executable files for efficient malware detection in IoT," Futur. Gener. Comput. Syst., 2019, doi: 10.1016/j.future.2019.04.044.

[37] H. S. Ham, H. H. Kim, M. S. Kim, and M. J. Choi, "Linear SVM-based Android malware detection for reliable IoT services," J. Appl. Math., vol. 2014, 2014, doi: 10.1155/2014/594501. 
[38] Z. Ren, H. Wu, Q. Ning, I. Hussain, and B. Chen, "End-to-end malware detection for android IoT devices using deep learning," Ad Hoc Networks, vol. 101, p. 102098, 2020, doi: 10.1016/j.adhoc.2020.102098.

[39] H. T. Nguyen, Q. D. Ngo, D. H. Nguyen, and V. H. Le, "PSI-rooted subgraph: A novel feature for IoT botnet detection using classifier algorithms," ICT Express, no. xxxx, 2020, doi: 10.1016/j.icte.2019.12.001.

[40] W. Jung, H. Zhao, M. Sun, and G. Zhou, "IoT botnet detection via power consumption modeling," Smart Heal., vol. 15, p. 100103, 2020, doi: 10.1016/j.smhl.2019.100103.

[41] H. El Merabet and A. Hajraoui, "A survey of malware detection techniques based on machine learning," Int. J. Adv. Comput. Sci. Appl., vol. 10, no. 1, pp. 366-373, 2019, doi: 10.14569/IJACSA.2019.0100148.

[42] D. Ucci, L. Aniello, and R. Baldoni, "Survey of machine learning techniques for malware analysis," Comput. Secur., vol. 81, pp. 123-147, 2019, doi: 10.1016/j.cose.2018.11.001.

[43] R. Sihwail, K. Omar, and K. A. Z. Ariffin, "A survey on malware analysis techniques: Static, dynamic, hybrid and memory analysis," Int. J. Adv. Sci. Eng. Inf. Technol., vol. 8, no. 4-2, pp. 1662-1671, 2018, doi: 10.18517/ijaseit.8.4-2.6827.

[44] J. Landage and M. Wankhade, "Malware and Malware Detection Techniques: A Survey," Int. J. Eng. Res. Technol., vol. 2, no. 12, pp. 61-68, 2013.

[45] S. Talukder, "Tools and Techniques for Malware Detection and Analysis," ArXiv, 2020.

[46] K. Diaz-Chito, F. J. Ferri, and A. Hernández-Sabaté, "An overview of incremental feature extraction methods based on linear subspaces," Knowledge-Based Syst., vol. 145, pp. 1-14, 2018, doi: 10.1016/j.knosys.2018.01.020.

[47] M. F. Rafique, M. Ali, J. Y. K. Qureshi, Aqsa Saeed, Asifullah Khan, and and A. M. Mirza, "Malware Classification using Deep Learning based Feature Extraction and Wrapper based Feature Selection Technique," ArXiv, pp. 1-20, 2019.

[48] M. A. Al-Garadi, A. Mohamed, A. Al-Ali, X. Du, and M. Guizani, "A Survey of Machine and Deep Learning Methods for Internet of Things (IoT) Security,” ACM Comput. Surv., vol. 5, pp. 1-42, 2018.

[49] S. M. Tahsien, H. Karimipour, and P. Spachos, "Machine learning based solutions for security of Internet of Things (IoT): A survey,” J. Netw. Comput. Appl., vol. 161, no. April, 2020, doi: 10.1016/j.jnca.2020.102630.

[50] M. Egele, T. Scholte, E. Kirda, and C. Kruegel, "A survey on automated dynamic malware-analysis techniques and tools,” ACM Comput. Surv., vol. 44, no. 2, pp. 1-49, 2012, doi: 10.1145/2089125.2089126.

\section{BIOGRAPHIES OF AUTHORS}

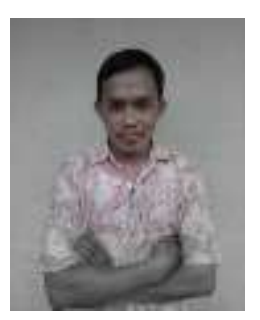

Susanto received his master degree in Computer Science from Universitas Bina Darma Palembang, South Sumatera, Indonesia. Currently he is a PhD candidate at Faculty of Engineering, Universitas Sriwijaya. He is currently a senior lecturer at Faculty of Computer, Universitas Bina Insan, Indonesia. His research interests include cryptography, information technology, information security, and network security.

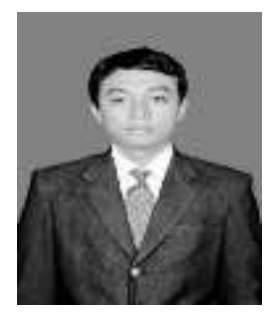

M. Agus Syamsul Arifin received his master degree in Computer Science from Universitas Bina Darma Palembang, South Sumatera, Indonesia. Currently he is a $\mathrm{PhD}$ candidate at Faculty of Engineering, Universitas Sriwijaya. He is currently a senior lecturer at Faculty of Computer, Universitas Bina Insan, Indonesia. His research interests include information technology, information security, and network security.

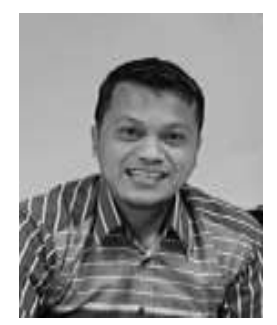

Deris Stiawan received the $\mathrm{PhD}$ degree in Computer Engineering from Universiti Teknologi Malaysia, Malaysia. He is currently an Associate Professor at Department of Computer Engineering, Faculty of Computer Science, Universitas Sriwijaya. His research interests include computer network, Intrusion Detection/Prevention System, and heterogeneous network 


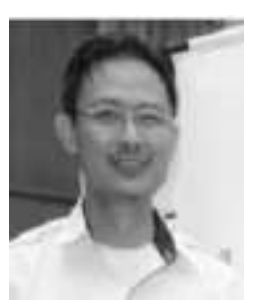

Mohd Yazid Idris is an Associate Professor at School of Computing, Faculty of Engineering, Universiti Teknologi Malaysia. He obtained his M.Sc and Ph.D. in the area of Software Engineering, and Information Technology (IT) Security in 1998 and 2008 respectively. In software engineering, he focuses on the research of designing and development of mobile and telecommunication software. His main research activity in IT security is in the area of Intrusion Prevention and Detection (IPD).

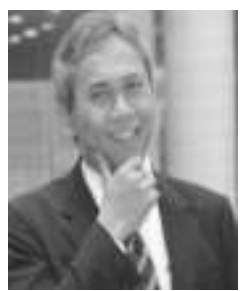

Rahmat Budiarto received B.Sc. degree from Bandung Institute of Technology in 1986, M.Eng. and Dr.Eng. in Computer Science from Nagoya Institute of Technology in 1995 and 1998, respectively. Currently, he is a full Professor at College of Computer Science and IT, Albaha University, Saudi Arabia. His research interests include intelligent systems, brain modeling, IPv6, network security, Wireless sensor networks, and MANETs. 